[6] P. de.la $\mathrm{H}$ a r pe, Classical Banach-Lie algebras and Banach-Lie groups of operators in Hilbert space, Lecture Notes in Math., No. 285, Springer Verlag, 1972.

[7] A. A. K i ri $110 \mathrm{v}$, Representations of the infinite-dimensional unitary group (Russian), Dokl. Akad. Nauk SSSR 212 (1973), 288-290.

[8] W. Krieger, On constructing non-isomorphic hyperfinite factors of type III, J. Func. Analysis 6 (1970), 97-109.

[9] -, On a dimension for a class of homeomorphism groups, preprint, 1976.

[10] $\mathrm{A}$. Li e b e rmann, The structure of certain unitary representations of infinite symmetric group, Trans. Amer. Math. Soc. 164 (1972), 189-198.

[11] J. von $\mathrm{N}$ e u m a n $\mathrm{n}$, On infinite direct products, Compositio Math. 6 (1938), 1-77.

[12] R. J. P 1 y m e n, Recent results on infinite-dimensional spin-groups, Adv. Math., to appear

[13] R. T. P o w e r s and E. S $ø \mathrm{r} \mathrm{mer}$, Free states of the canonical anticommutation relations, Commun. Math. Phys. 16 (1970), 1-33.

[14] I. E. S e g a 1, Tensor algebras over Hilbert spaces, I, Trans. Amer. Math. Soc. 81 (1956), 106-134; II, Annals of Math. 63 (1956), 160-175.

[15] - The structure of a class of representations of the unitary group on a Hilbert space, Proc. Amer. Math. Soc. 8 (1957), 197-203.

[16] Ş. S tră ti 1 ă et D. Vo i cul e s cu, Sur les représentations factorielles infinies de $U(\infty)$, C. R. Acad. Sc. Paris 280 (1975), 555-558.

[17] -, -, Representations of AF-algebras and of the group $U(\infty)$, Lecture Notes in Math., No. 486, Springer Verlag, 1975.

[18] - - On a class of KMS-states for the unitary group U( $\infty$ ), INCREST Preprint No. 23/1977.

[19] E. T h o ma, Die unzerlegbaren, positiv-definitien Klassenfunktionen der abzählbar unendlichen, symmetrischen Gruppe, Math. Z. 85 (1974), 40-61.

[20] - Characters of the group $\mathrm{GL}(\infty, q)$, in Conference on Harmonic Analysis, College Park, Maryland, 1971, Lecture Notes in Math., No. 266, Springer Verlag, 1972, 321-323.

[21] D. Vo iculescu, Sur les représentations factorielles finies de $U(\infty)$ et autres groupes semblables, C. R. Acad. Sc. Paris 279 (1974), 945-946.

[22] -, Représentations factorielles de type $\mathrm{II}_{1}$ de $U(\infty)$, J. Math. Pures Appl. 55 (1976), 1-20.

[23] H. W e y 1, The classical groups. Their invariants and representations, Princeton University Press, 1939.

[24] D. P. Ž helobenko, Compact Lie groups and their representations (Russian), Nauka, Moscow 1970.

Presented to the semester

Spectral Theory

September 23-December 16, 1977
BANACH CENTER PUBLICATIONS, VOLUME 8

PWN-POLISH SCIENTIFIC PUBLISHERS

WARSAW 1982

\section{LINEAR PREDICTOR FOR STATIONARY PROCESSES IN COMPLETE CORRELATED ACTIONS}

\author{
ION SUCIU and ILIE VALUSESCU
}

Department of Mathematics, The National Institute for Scientific and Technical Creation, Bucharest, România

\section{Introduction}

In this paper we shall continue the study of prediction theory of a stationary process considered as time evolution in a correlated action, which was began in [4]. As in the precedent paper, we shall follow the line of Wiener and Masani prediction schema for (finite) multivariate stationary process [7], [8].

The notion of completion of a correlated action, which we shall introduce in Section 2, will allow us to give a precise meaning to the predictible part of the process and, consequently, to formulate more precisely the prediction problems (Section 3). Since some results from [4] are used here in a slightly different context, we prefer to outline their proofs. In Section 4, under the supplementary condition of boundedness imposed on the spectral distribution of the process, similar to Wiener-Masani boundedness condition [8], we shall determine the predictible part of the process by means of a linear (infinite) Wiener filter. The solution of prediction problems are given in terms of Taylor coefficients of the maximal outer function which factorizes the spectral distribution of the process (see [3]).

The reader will certainly note that we permanently use the ideas from the Sz.-Nagy and C. Foiaș model for contraction [6] to give an operator or functional model for prediction. Our model is based on an operator valued positive definite map (on the integers), which corresponds to an infinite variate (discrete) stationary process.

\section{Complete correlated actions}

The notion of correlated action was introduced in [4] as the triplet $\{\mathscr{E}, \mathscr{H}, \Gamma\}$, where $\mathscr{E}$ is a Hilbert space (the space of the parameters), $\mathscr{H}$ is a right $\mathscr{L}(\mathscr{E})$-module (the state space), and $\Gamma: \mathscr{H} \times \mathscr{H} \rightarrow \mathscr{L}(\mathscr{E})$ is an $\mathscr{L}(\mathscr{E})$-valued map (the correlation) with the properties:

$$
\text { (i) } \Gamma[h, h] \geqslant 0, \Gamma[h, h]=0 \Rightarrow h=0 \text {. }
$$


(ii) $\Gamma\left[h_{1}, h_{2}\right]=\Gamma\left(h_{2}, h_{1}\right]^{*}$.

(iii) $\Gamma\left[\sum_{i} A_{i} h_{i}, \sum_{j} B_{j} g_{j}\right]=\sum_{i, j} A_{i}^{*} \Gamma\left[h_{i}, g_{j}\right] B_{j}$.

Let now $\mathscr{E}, \mathscr{K}$ be two Hilbert spaces and $\mathscr{H}=\mathscr{L}(\mathscr{E}, \mathscr{K})$. Putting for $A$ $\in \mathscr{L}(\mathscr{E})$ and $V \in \mathscr{L}(\mathscr{E}, \mathscr{K})$

$$
A V=V A
$$

where $V A$ is the usual composition of operators, we make $\mathscr{H}$ a right $\mathscr{L}(\mathscr{E})$-module. If we define $T$ by

$$
\text { (2.1) } \quad I\left[V_{1}, V_{2}\right]=V_{1}^{*} V_{2} \text {, }
$$

then obviously $\Gamma$ satisfies properties (i) and (ii). (iii) is also fulfilled:

$$
\begin{aligned}
\Gamma\left[\sum_{i} A_{i} V_{i}, \sum_{j} B_{j} W_{j}\right] & =\left(\sum_{i} V_{i} A_{i}\right)^{*}\left(\sum_{j} W_{j} B_{j}\right) \\
& =\sum_{i, j} A_{i}^{*} V_{i}^{*} W_{j} B_{j}=\sum_{i, j} A_{i}^{*} \Gamma\left[V_{i}, W_{j}\right] B_{j} .
\end{aligned}
$$

Hence $\{\mathscr{E}, \mathscr{H}, \Gamma\}$ is a correlated action. In fact, as the following proposition shows, any correlated action can be imbedded into one of this type.

Proposition 1. Let $\{\mathscr{E}, \mathscr{H}, \Gamma\}$ be a correlated action. There exist a Hilbert space $\mathscr{K}$ and an algebraic imbedding $h \rightarrow V_{h}$ of the right $\mathscr{L}(\mathscr{E})$-module $\mathscr{H}$ into the right $\mathscr{L}(\mathscr{E})$-module $\mathscr{L}(\mathscr{E}, \mathscr{K})$ with the properties

$$
\Gamma\left[h_{1}, h_{2}\right]=V_{h_{1}}^{*} V_{h_{2}}, \quad h_{1}, h_{2} \in \mathscr{H} .
$$

(2.3) The elements of the form $\gamma_{(a, h)}=V_{h} a$, with $a \in \mathscr{E}$ and $h \in \mathscr{H}$, span a dense subspace in $\mathscr{K}$.

This imbedding is unique up to a unitary equivalence.

Proof. The proof follows the construction of the Aronszajn reproducing kernel in a Hilbert space [1], [2]. Let $A=\mathscr{E} \times \mathscr{H}$ and $\gamma_{(a, h)}$ be the complex valued function defined on $A$ by

$$
\gamma_{(a, h)}(b, g)=(\Gamma[g, h] a, b)_{s} .
$$

On the linear span of these functions we define the form

$$
\left\langle\sum_{j} \gamma_{\left(a_{j}, h_{j}\right)}, \sum_{k} \gamma_{\left(b_{k}, g_{k}\right)}\right\rangle=\sum_{j, k}\left(\Gamma\left[g_{k}, h_{j}\right] a_{j}, b_{k}\right)_{\tilde{\delta}} .
$$

For $a_{1}, \ldots, a_{n} \in \mathscr{E}$, choose $a \in \mathscr{E}$ and $A_{j} \in \mathscr{L}(\mathscr{E})$ such that $A_{j} a=a_{j}$. We have

$$
\begin{aligned}
\left\langle\sum_{j} \gamma_{\left(a_{j}, h_{j}\right)}, \sum_{k} \gamma_{\left(a_{k}, h_{k}\right)}\right\rangle & =\sum_{j, k}\left(\Gamma\left[h_{k}, h_{j}\right] a_{j}, a_{k}\right)_{\delta} \\
& =\sum_{j, k}\left(\Gamma\left[h_{k}, h_{j}\right] A_{j} a, A_{k} a\right)_{\delta}=\sum_{j, k}\left(A_{k}^{*} \Gamma\left[h_{k}, h_{j}\right] A_{j} a, a\right)_{\delta} \\
& =\left(\Gamma\left[\sum_{k} A_{k} h_{k}, \sum_{j} A_{j} h_{j}\right] a, a\right) \geqslant 0 .
\end{aligned}
$$

Thus $\langle\cdot, \cdot\rangle$ is a sesquilinear semi-positive definite form. The Hilbert space $\mathscr{K}$ is obtained in the usual way from this form.

For any $h \in \mathscr{H}$ we define

$$
V_{h} a=\gamma_{(a, h),} \quad a \in \mathscr{E} .
$$

Using (2.5) and (2.4) we have.

$$
\left\|V_{h} a\right\|_{\mathscr{C}}^{2}=\left\|\gamma_{(a, h)}\right\|_{\mathscr{C}}^{2}=(\Gamma[h, h] a, a)_{\mathcal{E}} \leqslant\|\Gamma[h, h]\| \cdot\|a\|^{2} ;
$$

therefore $V_{h} \in \mathscr{L}(\mathscr{E}, \mathscr{K})$.

For any $h_{1}, h_{2} \in \mathscr{H}$ we have

$$
\left(\Gamma\left[h_{1}, h_{2}\right] a, b\right)_{\&}=\left\langle\gamma_{\left(a, h_{2}\right)}, \gamma_{\left(b, h_{1}\right)}\right\rangle=\left\langle V_{h_{2}} a, V_{h_{1}} b\right\rangle=\left(V_{h_{1}}^{*} V_{h_{2}} a, b\right)_{\&} .
$$

Hence

$$
\Gamma\left[h_{1}, h_{2}\right]=V_{h_{1}}^{*} V_{h_{2}}
$$

and so property (2.2) is verified. Property (2.3) results from the construction of the Hilbert space $\mathscr{K}$.

If $h \rightarrow V_{h}^{\prime}$ is another imbedding of $\mathscr{H}$ into $\mathscr{L}\left(\mathscr{E}, \mathscr{K}^{\prime}\right)$ which verifies (2.2) and (2.3), then setting

$$
X V_{h}^{\prime} a=V_{h} a
$$

we obtain a unitary operator $X: \mathscr{K}^{\prime} \rightarrow \mathscr{K}$ such that

$$
X V_{h}^{\prime}=V_{h} \text {. }
$$

The proof of the proposition is finished.

The Hilbert space $\mathscr{K}$, uniquely attached to $\{\mathscr{E}, \mathscr{H}, \Gamma\}$ as in Proposition 1 , is called the measuring space of the correlated action $\{\mathscr{E}, \mathscr{H}, \Gamma\}$.

We say that $\{\mathscr{E}, \mathscr{H}, \Gamma\}$ is a complete correlated action, if the map $h \rightarrow V_{h}$ of $\mathscr{H}$ into $\mathscr{L}(\mathscr{E}, \mathscr{K})$ is onto.

Recall that a $\Gamma$-stationary (discrete) process in a correlated action $\{\mathscr{E}, \mathscr{H}, \Gamma\}$ is a sequence $\left\{f_{n}\right\}_{-\infty}^{+\infty}$ of elements in $\mathscr{H}$ such that $\Gamma\left[f_{n}, f_{m}\right]$ depends only of the difference $m-n$ and not on $m$ and $n$ separately.

For a $\Gamma$-stationary process $\left\{f_{n}\right\}_{-\infty}^{+\infty}$ we use the following notation:

Remark that we also have

$$
\begin{gathered}
\mathscr{H}_{n}^{S}=\left\{h \in \mathscr{H} \mid h=\sum_{i<n} A_{i} f_{t}, A_{i} \in \mathscr{L}(\mathscr{E})\right\}, \\
\mathscr{K}_{n}^{f}=\bigvee_{k=-\infty}^{n} V_{f_{k}} \mathscr{E}, \quad \mathscr{K}_{\infty}^{S}=\bigvee_{-\infty}^{+\infty} V_{f_{k}} \mathscr{E} .
\end{gathered}
$$

$$
\mathscr{K}_{n}^{s}=\bigvee_{n \in \mathscr{X}_{n}^{\prime}} V_{n} \mathscr{E}
$$

We say that two $\Gamma$-stationary processes $\left\{f_{n}\right\}_{-\infty}^{+\infty}$ and $\left\{g_{n}\right\}_{-\infty}^{+\infty}$ are stationarily cross-correlated if $\Gamma\left[f_{n}, g_{m}\right]$ depends only of the difference $m-n$.

PROPOsITION 2. For any $\Gamma$-stationary process $\left\{f_{n}\right\}_{-\infty}^{+\infty}$ there exists a unitary operator $U_{f}$ on $\mathscr{K}_{\infty}^{f}$ such that

$$
V_{f_{n}}=U_{f}^{n} V_{s_{0}}
$$


The $\Gamma$-stationary process $\left\{g_{n}\right\}_{-\infty}^{+\infty}$ is stationary cross-correlated with $\left\{f_{n}\right\}_{-\infty}^{+\infty}$ iff there exists a unitary operator $U_{f g}$ on

such that

$$
\mathscr{K}_{\infty}^{f g}=\mathscr{K}_{\infty}^{f} \vee \mathscr{K}_{\infty}^{g}
$$

Proof. Setting on the generators of $\mathscr{K}_{\infty}^{s}$

$$
U_{f} V_{f_{n}} a=V_{f_{n+1}} a,
$$

clearly we obtain a unitary operator on $\mathscr{K}_{\infty}^{f}$ satisfying (2.6).

Let $\left\{f_{n}\right\}_{-\infty}^{+\infty}$ and $\left\{g_{n}\right\}_{-\infty}^{+\infty}$ be stationary cross-correlated processes, and $U_{f}, U_{g}$ be as above. Then, if we put

$$
U_{f g}\left(V_{f_{n}} a+V_{g_{m}} b\right)=V_{f_{n+1}} a+V_{g_{m+1}} b
$$

we have

$$
\begin{aligned}
\left.\left\|U_{f g}\left(V_{s_{n}} a+V_{g_{m}} b\right)\right\|\right|^{2}= & \left\|V_{f_{n+1}} a+V_{g_{m+1}} b\right\|^{2}=\left\|\gamma_{\left(a, f_{n+1}\right.}+\gamma_{\left(b, g_{m+1}\right)}\right\|^{2} \\
= & \left\langle\gamma_{\left(a, f_{n+1}\right)}+\gamma_{\left(b, g_{m+1}\right)}, \gamma_{\left(a, f_{n+1}\right)}+\gamma_{\left(b, g_{m+1}\right)}\right\rangle \\
= & \left(\Gamma\left[f_{n+1}, f_{n+1}\right] a, a\right)+\left(\Gamma\left[g_{m+1}, g_{m+1}\right] b, b\right)+ \\
& +2 \operatorname{Re}\left(\Gamma\left[f_{n+1}, g_{m+1}\right] b, a\right) \\
= & \left(\Gamma\left[f_{n}, f_{n}\right] a, a\right)+\left(\Gamma\left[g_{m}, g_{m}\right] b, b\right)+2 \operatorname{Re}\left(\Gamma\left[f_{n}, g_{m}\right] b, a\right) \\
= & \ldots=\left\|V_{f_{n}} a+V_{g_{m}} b\right\|^{2} .
\end{aligned}
$$

It follows that (2.7) defines a unitary operator $U_{f g}$ on $\mathscr{K}_{\infty}^{f g}$ which extends both $U_{f}$ and $U_{g}$.

The unitary operator $U_{f}$ is called the shift operator attached to the $\Gamma$-stationary process $\left\{f_{n}\right\}_{-\infty}^{+\infty}$ and $U_{f 8}$ the extended shift of the stationary cross-correlated processes $\left\{f_{n}\right\}_{-\infty}^{+\infty}$ and $\left\{g_{n}\right\}_{-\infty}^{+\infty}$.

Let us remark that (2.6) implies

$$
\mathscr{K}_{\infty}^{f}=\bigvee_{-\infty}^{+\infty} U_{f}^{n} V_{f} \mathscr{E}
$$

where $V_{f}=V_{f_{0}}$.

In what follows, we use the following notation:

$$
\mathscr{K}_{+}=\bigvee_{0}^{\infty} U_{f}^{* n} V_{f} \mathscr{E}=\mathscr{K}_{0}
$$

A $\Gamma$-stationary process $\left\{g_{n}\right\}_{-\infty}^{+\infty}$ is called a white noise process, provided $\Gamma\left[g_{n}, g_{m}\right]=0$ for $n \neq m$.

We say that a process $\left\{f_{n}\right\}_{-\infty}^{+\infty}$ contains a white noise process $\left\{g_{n}\right\}_{-\infty}^{+\infty}$ if:

(i) $\left\{g_{n}\right\}_{-\infty}^{+\infty}$ is stationary cross-correlated with $\left\{f_{n}\right\}_{-\infty}^{+\infty}$ and

$$
\Gamma\left[f_{n}, g_{m}\right]=0, \quad m>n,
$$

(ii) $V_{g} \mathscr{E} \subset \mathscr{K}_{+}^{f}$,

(iii) $\operatorname{Re} \Gamma\left[f_{n}-g_{n}, g_{n}\right] \geqslant 0$

A $\Gamma$-stationary process $\left\{f_{n}\right\}_{-\infty}^{+\infty}$ is called deterministic if it contains no nonzero white noise process.

We say that a $\Gamma$-stationary process $\left\{f_{n}\right\}_{-\infty}^{+\infty}$ is a moving average of a white noise $\left\{g_{n}\right\}_{-\infty}^{+\infty}$ if $\left\{f_{n}\right\}_{-\infty}^{+\infty}$ contains $\left\{g_{n}\right\}_{-\infty}^{+\infty}$ and $\mathscr{K}_{\infty}^{g}=\mathscr{K}_{\infty}^{f}$.

THeorem 1. (Wold decomposition in time domain.) A $I$-stationary process $\left\{f_{n}\right\}_{-\infty}^{+\infty}$ admits a unique decomposition of the form

$$
f_{n}=u_{n}+v_{n}
$$

where $\left\{u_{n}\right\}_{-\infty}^{+\infty}$ is a moving average of a white noise $\left\{g_{n}\right\}_{-\infty}^{+\infty}$ contained in $\left\{f_{n}\right\}_{-\infty}^{+\infty}$, $\left\{v_{n}\right\}_{-\infty}^{+\infty}$ is a deterministic process, and $\Gamma\left[u_{n}, v_{m}\right]=0$ for any $n, m$. The white noise $\left\{g_{n}\right\}_{-\infty}^{+\infty}$ is the maximal white noise process contained in $\left\{f_{n}\right\}_{-\infty}^{+\infty}$.

Proof. Using the imbedding $h \rightarrow V_{h}$ of $\mathscr{H}$ into $\mathscr{L}(\mathscr{E}, \mathscr{K})$ and (2.6), we may consider

$$
f_{n}=U_{f}^{n} V_{f} .
$$

By the Wold decomposition of the isometric operator $U_{+}$on $\mathscr{K}_{+}$, we have

$$
\mathscr{K}_{+}=M_{+}(\mathscr{F}) \oplus \mathscr{R}
$$

where

$$
\mathscr{F}=\mathscr{K}_{+} \ominus U_{+} \mathscr{K}_{+}, \quad M_{+}(\mathscr{F})=\bigoplus_{0}^{\infty} U_{+}^{n} \mathscr{F} \quad \text { and } \quad \mathscr{R}=\bigcap_{n \geqslant 0} U_{+}^{n} \mathscr{K}_{+} .
$$

Let $P$ be the orthogonal projection of $\mathscr{K}_{+}$onto $M_{+}(\mathscr{F})$ and $P_{\mathscr{F}}$ be the orthogonal projection of $\mathscr{K}_{+}$on the wandering subspace $\mathscr{F}$. If we put $u_{n}=U_{f}^{n} P V_{f}$, $v_{n}=U_{f}^{n}(I-P) V_{f}$ and $g_{n}=U_{f}^{n} P_{\mathscr{F}} V_{f}$, then (2.10) is obvious and we have

Since

$$
\Gamma\left[u_{n}, v_{n}\right]=V_{f}^{*} P U_{f}^{m-n}(I-P) V_{f}=V_{f}^{*} U_{f}^{m-n} P(I-P) V_{f}=0 .
$$

$$
\Gamma\left[g_{n}, g_{m}\right]=V_{f}^{*} P_{\mathscr{F}} U_{f}^{m-n} P_{\mathscr{F}} V_{f}=0, \quad n \neq m,
$$

it follows that $\left\{g_{n}\right\}_{-\infty}^{+\infty}$ is a white noise process. The $T$-stationary white noise process $\left\{g_{n}\right\}_{-\infty}^{+\infty}$ is contained in $\left\{u_{n}\right\}_{-\infty}^{+\infty}$. Indeed, we have:

(i) $\left\{g_{n}\right\}_{-\infty}^{+\infty}$ is stationary cross-correlated with $\left\{u_{n}\right\}_{-\infty}^{+\infty}$ and

$$
\Gamma\left[u_{n}, g_{m}\right]=V_{f}^{*} P U_{f}^{m-n} P_{\mathscr{F}} V_{f}=0 \text { for } m>n .
$$

(ii) $V_{g} \mathscr{E}=P_{\mathscr{F}} V_{f} \mathscr{E} \subset P V_{f} \mathscr{E} \subset \mathscr{K}_{+}^{u}$.

(iii) $\Gamma\left[u_{n}-g_{n}, g_{n}\right]=\Gamma\left[u_{n}, g_{n}\right]-\Gamma\left[g_{n}, g_{n}\right]=V_{f}^{*} P P_{\mathscr{F}} V_{f}-V_{f}^{*} P_{\mathscr{F}} V_{f}=0$

Since we clearly have

$$
\mathscr{K}_{\infty}^{g}=\mathscr{K}_{\infty}^{u}=M(\mathscr{F}),
$$

it follows that the process $\left\{u_{n}\right\}_{-\infty}^{+\infty}$ is a moving average of the white noise process $\left\{g_{n}\right\}_{-\infty}^{+\infty}$.

Let us check that the white noise $\left\{g_{n}\right\}_{-\infty}^{+\infty}$ is also contained in the $\Gamma$-stationary process $\left\{f_{n}\right\}_{-\infty}^{+\infty}$ : 
(1) For any $a \in \mathscr{E}$ and $m>n$ we have

$$
\left(\Gamma\left[f_{n}, g_{m}\right] a, a\right)_{\mathscr{\delta}}=\left(V_{f}^{*} U_{f}^{m-n} P_{\mathscr{F}} V_{f} a, a\right)_{\mathscr{E}}=\left(P_{\mathscr{F}} V_{\mathscr{F}} a, U_{+}^{m-n} V_{f} a\right)_{\mathscr{E}}=0 .
$$

We have also:

(2) $V_{g} \mathscr{E}=P_{\mathscr{F}} V_{f} \mathscr{E} \subset \mathscr{K}_{+}^{s}$,

(3) $\Gamma\left[f_{n}-g_{n}, g_{n}\right]=\Gamma\left[f_{n}, g_{n}\right]-\Gamma\left[g_{n}, g_{n}\right]=V_{f}^{*} P_{\mathscr{F}} V_{f}-V_{f}^{*} P_{\mathscr{F}}^{z} V_{f}=0$

Hence the white noise $\left\{g_{n}\right\}_{-\infty}^{+\infty}$ is contained in $\left\{f_{n}\right\}_{-\infty}^{+\infty}$.

Let $\left\{g_{n}^{\prime}\right\}_{-\infty}^{+\infty}$ be another white noise process contained in $\left\{f_{n}\right\}_{-\infty}^{+\infty}$. We shall see that $\left\{\boldsymbol{g}_{n}^{\prime}\right\}_{-\infty}^{+\infty}$ is contained in $\left\{g_{n}\right\}_{-\infty}^{+\infty}$, too. Firstly, we see that

$$
V_{g^{\prime}, \mathscr{E}} \subset \mathscr{F} \text {. }
$$

Indeed, remarking that the extended shift $U_{f \xi^{\prime}}$ equals $U_{f}$, for any $a, a_{n} \in \mathscr{E}$ we have

$$
\left(V_{g^{\prime}} a, U_{f}^{*+1} V_{f} a_{n}\right)_{\mathscr{C}}=\left(V_{f}^{*} U_{f}^{n+1} V_{g^{\prime}} a, a_{n}\right)_{s}=\left(\Gamma\left[f_{0}, g_{n+1}^{\prime}\right] a, a_{n}\right)=0,
$$

because $\left\{g_{n}^{\prime}\right\}_{-\infty}^{+\infty}$ is contained in $\left\{f_{n}\right\}_{-\infty}^{+\infty}$. Therefore

$$
V_{g^{\prime}, \mathscr{E}} \subset \mathscr{F} \subset M_{+}(\mathscr{F F})=\mathscr{K}_{+}^{g} .
$$

From (2.14) it is seen that for $m>n$ we have

Since

$$
\Gamma\left[g_{n}, g_{n}^{\prime}\right]=V_{f}^{*} P_{\mathscr{F}} U_{f}^{m-n} V_{g^{\prime}}=0 .
$$

$\Gamma\left[g_{n}-g_{n}^{\prime}, g_{n}^{\prime}\right]=\Gamma\left[g_{n}, g_{n}^{\prime}\right]-\Gamma\left[g_{n}^{\prime}, g_{n}^{\prime}\right]=V_{f}^{*} P_{\mathscr{F}} V_{g^{\prime}}-\Gamma\left[g_{n}^{\prime}, g_{n}^{\prime}\right]$

$$
=V_{s}^{*} V_{g^{\prime}}-\Gamma\left[g_{n}^{\prime}, g_{n}^{\prime}\right]=\Gamma\left[f_{n}, g_{n}^{\prime}\right]-\Gamma\left[g_{n}^{\prime}, g_{n}^{\prime}\right]=\Gamma\left[f_{n}-g_{n}^{\prime}, g_{n}^{\prime}\right],
$$

it results $\left(\left\{g_{n}^{\prime}\right\}_{-\infty}^{+\infty}\right.$ being contained in $\left.\left\{f_{n}\right\}_{-\infty}^{+\infty}\right)$ that $\operatorname{Re} \Gamma\left[g_{n}-g_{n}^{\prime}, g_{n}^{\prime}\right] \geqslant 0$. Hence $\left\{g_{n}^{\prime}\right\}_{-\infty}^{+\infty}$ is contained in $\left\{g_{n}\right\}_{-\infty}^{+\infty}$, i.e. $\left\{g_{n}\right\}_{-\infty}^{+\infty}$ is the maximal white noise contained in $\left\{f_{n}\right\}_{-\infty}^{+\infty}$.

Let $\left\{l_{n}\right\}_{-\infty}^{+\infty}$ be a white noise contained in $\left\{v_{n}\right\}_{-\infty}^{+\infty}$. Then we have

$$
\Gamma\left[u_{n}, l_{m}\right]=V_{f}^{*} P U_{f}^{m-n} V_{l}=V_{f}^{*} P U_{f}^{m-n}(I-P) V_{l}=0 .
$$

It follows that $\left\{l_{n}\right\}_{-\infty}^{+\infty}$ and $\left\{f_{n}\right\}_{-\infty}^{+\infty}$ are cross-correlated and $\Gamma\left[f_{n}, l_{m}\right]=0$.

The fact that $V_{l} \mathscr{E} \subset \mathscr{K}_{+}^{f}$ is obvious, and

$$
\operatorname{Re} \Gamma\left[f_{n}-l_{n}, l_{n}\right]=\operatorname{Re} \Gamma\left[u_{n}, l_{n}\right]+\operatorname{Re} \Gamma\left[v_{n}-l_{n}, l_{n}\right] \geqslant 0 .
$$

Therefore the white noise $\left\{l_{n}\right\}_{-\infty}^{+\infty}$ is contained in $\left\{f_{n}\right\}_{-\infty}^{+\infty}$, and by the maximality of $\left\{g_{n}\right\}_{-\infty}^{+\infty}$ in $\left\{f_{n}\right\}_{-\infty}^{+\infty}$ it follows that $\left\{l_{n}\right\}_{-\infty}^{+\infty}$ is contained in $\left\{g_{n}\right\}_{-\infty}^{+\infty}$. We then have

Hence

$$
\Gamma\left[g_{n}, l_{n}\right]=V_{f}^{*} P_{\mathcal{F}} V_{l}=V_{f}^{*} P_{\mathscr{F}}(I-P) V_{l}=0 .
$$

which implies $l_{n}=0$.

$$
\Gamma\left[l_{n}, l_{n}\right]=\operatorname{Re} \Gamma\left[g_{n}, l_{n}\right]-\operatorname{Re} \Gamma\left[g_{n}-l_{n}, l_{n}\right] \leqslant 0,
$$

If we consider

$$
f_{n}=u_{n}^{\prime}+v_{n}^{\prime}
$$

being another decomposition of the form (2.10) and if $\left\{u_{n}^{\prime}\right\}_{-\infty}^{+\infty}$ is a moving average of the white noise $\left\{g_{n}^{\prime}\right\}_{-\infty}^{+\infty}$ contained in $\left\{f_{n}\right\}_{-\infty}^{+\infty}$, then, by the maximality of $\left\{g_{n}\right\}_{-\infty}^{+\infty}$, it follows that $\left\{g_{n}^{\prime}\right\}_{-\infty}^{+\infty}$ is contained in $\left\{g_{n}\right\}_{-\infty}^{+\infty}$. Hence $V_{g^{\prime}} \mathscr{E} \subset \mathscr{K}_{+}^{g}=M_{+}(\mathscr{F})$. Moreover, $V_{g^{\prime}} \mathscr{E} \subset \mathscr{F}$. Indeed,

$$
\left(V_{g^{\prime}} a, U_{f}^{* n+1} V_{f} a_{n}\right)_{\mathcal{X}}=\left(V_{f}^{*} U_{f}^{n+1} V_{g^{\prime}}, a, a_{n}\right)_{\varepsilon}=\left(\Gamma\left[f_{0}, g_{n+1}^{\prime}\right] a, a_{n}\right)_{\varepsilon}=0
$$

and $V_{g}, \mathscr{E}$ is orthogonal on $U_{+} \mathscr{K}_{+}^{\text {s. }}$, i.e. $V_{g}, \mathscr{E} \subset \mathscr{F}$.

From (2.13) we have

and

$$
V_{f}=V_{u^{\prime}}+V_{v^{\prime}}
$$

$$
\mathscr{K}_{\infty}^{f}=\mathscr{K}_{\infty}^{u \prime} \oplus \mathscr{K}_{\infty}^{v^{\prime \prime}} .
$$

Let us denote: $\mathscr{F}_{1}=\mathscr{F} \ominus \overline{V_{g^{\prime}} \mathscr{E}}$ and $q_{n}=U_{f}^{n} P_{\mathscr{F}_{1}} V_{S}$. Then it is obvious that $\left\{q_{n}\right\}_{-\infty}^{+\infty}$ is a white noise process contained in $\left\{f_{n}\right\}_{-\infty}^{+\infty}$ and we have:

(i) $\left(\Gamma\left[v_{n}^{\prime}, q_{m}\right] a, a\right)=\left(V_{v^{\prime}}^{*} U_{f}^{m-n} P_{\mathscr{F}_{1}} V_{f} a, a\right)=\left(P_{\mathscr{F}_{1}} V_{f} a, U_{f}^{* m-n} V_{v^{\prime}} a\right)=0$,

(ii) $V_{q} \mathscr{E} \subset \mathscr{K}_{+}^{v^{\prime}}$ (by the fact that $V_{q} \mathscr{E} \perp \mathscr{K}_{+}^{u}$ and (2.16)),

(iii) $\operatorname{Re} \Gamma\left[v_{n}^{\prime}-q_{n}, q_{n}\right]=\operatorname{Re} \Gamma\left[v_{n}^{\prime}, q_{n}\right]-\Gamma\left[q_{n}, q_{n}\right]$

$$
=V_{v}^{*}, P_{\mathscr{F}_{1}} V_{f}-V_{f}^{*} P_{\mathscr{F}_{1}} V_{f}=V_{f}^{*} P_{\mathscr{F}_{1}} V_{f}-V_{f}^{*} P_{\mathscr{F}_{1}} V_{f}=0 .
$$

It results that the white noise process $\left\{q_{n}\right\}_{-\infty}^{+\infty}$ is contained in the deterministic process $\left\{v_{n}^{\prime}\right\}_{-\infty}^{+\infty}$, i.e. $q_{n}=0$. Therefore $\mathscr{F}_{1}=\{0\}$ and consequently $\overline{V_{g^{\prime}} \mathscr{E}}=\mathscr{F}$. Hence we obtain that $\mathscr{X}_{\infty}^{u \prime}=\mathscr{K}_{\infty}^{g^{\prime}}=M(\mathscr{F}), \mathscr{K}_{\infty}^{v^{\prime}}=\mathscr{R}$, and by (2.15), (2.16) it follows that $V_{u^{\prime}}=P V_{f}$. So we have $u^{\prime}=u$ and $v^{\prime}=v$.

The proof of the theorem is finished.

The process $\left\{g_{n}\right\}_{-\infty}^{+\infty}$ is the innovation part of the process $\left\{f_{n}\right\}_{-\infty}^{+\infty}$ and it is called the innovation-process associated with $\left\{f_{n}\right\}_{-\infty}^{+\infty}$.

\section{Prediction problems}

Let $\left\{f_{n}\right\}_{-\infty}^{+\infty}$ be a $\Gamma$-stationary process in a complete correlated action $\{\mathscr{E}, \mathscr{H}, \Gamma\}$. Denote:

$$
\mathscr{H}_{0}=\left\{h \in \mathscr{H} \mid h=\sum_{k \leqslant 0} A_{k} f_{k}, A_{k} \in \mathscr{L}(\mathscr{E})\right\}
$$

where only finitely many of the $A_{k}$ are non-zero operators. Following Wiener and Masani [7], we call $\mathscr{H}_{0}$ the present and past of the process $\left\{f_{n}\right\}_{-\infty}^{+\infty}$ and interprete it as the total information obtained on the process up to the present moment $(t=0)$.

To predict the process at the next moment $(t=1)$ means to obtain the best information about $f_{1}$ in terms of the elements in $\mathscr{H}_{0}$. The following proposition will give this a precise sense.

Proposition 3. Let $\left\{f_{n}\right\}_{-\infty}^{+\infty}$ be as $\Gamma$-stationary process and $\left\{g_{n}\right\}_{-\infty}^{+\infty}$ be the maximal white noise contained in it. Setting

$$
\hat{f}_{1}=f_{1}-g_{1},
$$


we have $\Gamma\left[\hat{f}_{1}, g_{1}\right]=0$ and

$$
\Gamma\left[f_{1}-\hat{f}_{1}, f_{1}-\hat{f}_{1}\right]=\inf _{h \in \mathscr{K}_{0}} \Gamma\left[f_{1}-h, f_{1}-h\right]
$$

where the infimum is taken in the set of the positive operators in $\mathscr{L}(\mathbb{E})$

For any $a \in \mathscr{E}$ we have

$$
\left(\Gamma\left[f_{1}-\hat{f}_{1}, f_{1}-\hat{f}_{1}\right] a, a\right)=\inf \sum_{j, k=0}^{m}\left(\Gamma\left[f_{j}, f_{k}\right] a_{j}, a_{k}\right)
$$

where the infimum is taken over all finite systems $a_{1}, \ldots, a_{m}$ in $\mathscr{E}$. and $a_{0}=a$.

Proof (see [4]). For any $a \in \mathscr{E}$ we have

$$
\begin{aligned}
& \left(\Gamma\left[f_{1}-\hat{f}_{1}, f_{1}-\hat{f}_{1}\right] a, a\right)_{\mathscr{G}}=\left(\Gamma\left[g_{1}, g_{1}\right] a, a\right)=\left(V_{f}^{*} P_{\mathscr{F}} V_{f} a, a\right)=\left\|P_{\mathscr{F}} V_{f} a\right\|^{2} \\
& =\inf _{k \in U_{+} \mathscr{C}_{+}}\left\|V_{f} a-k\right\|^{2}=\inf _{a_{1}, \ldots, a_{m} \in \Theta}\left\|V_{f} a+\sum_{k=1}^{m} U_{f}^{* k} V_{f} a_{k}\right\|^{2} \\
& =\inf \left\|\sum_{k=0}^{m} U_{f}^{* k} V_{f} a_{k}\right\|^{2}=\inf \sum_{k, j=0}^{m}\left(V_{f}^{*} U_{f}^{k-j} V_{f} a_{j}, a_{k}\right)_{f} \\
& =\inf _{\substack{a_{3}, \ldots, a_{m} \in a_{0} \\
a_{0}=a}} \sum_{k, j=0}^{m}\left(\Gamma\left[f_{j}, f_{k}\right] a_{j}, a_{k}\right)_{\&} ;
\end{aligned}
$$

thus (3.4) is proved.

Let now $h=\sum_{k=0}^{m} A_{k} f_{-k}$ be an arbitrary element in $\mathscr{H}_{0}$. For any $a \in \mathscr{E}$, setting $a_{k}=-A_{k} a$ we obtain

$$
\begin{aligned}
& \left(\Gamma\left[f_{1}-h, f_{1}-h\right] a, a\right)_{\delta}=\left(\Gamma\left[f_{1}-\sum_{k=0}^{m} A_{k} f_{-k}, f_{1}-\sum_{j=0}^{m} A_{j} f_{-j}\right] a, a\right)_{\delta} \\
& =\sum_{k=-1}^{m}\left(\Gamma\left[f_{-k}, f_{-j}\right] a_{j}, a_{k}\right)_{\delta}=\sum_{k=-1}^{m}\left(\Gamma\left[f_{j}, f_{k}\right] a_{j}, a_{k}\right)_{\delta}=\sum_{k=0}^{m+1}\left(\Gamma\left[f_{j}, f_{k}\right] a_{j}, a_{k}\right)_{\delta}
\end{aligned}
$$

From (3.4) it is clear that

$$
\Gamma\left[f_{1}-\hat{f}_{1}, f_{1}-\hat{f}_{1}\right] \leqslant \Gamma\left[f_{1}-h, f_{1}-h\right] .
$$

Let $A$ be a positive operator in $\mathscr{L}(\mathscr{E})$ such that for any $h \in \mathscr{H}_{0}$

$$
A \leqslant \Gamma\left[f_{1}-h, f_{1}-h\right] .
$$

For any $a \in \mathscr{\&}$ and $a_{1}, \ldots, a_{m} \in \mathscr{E}$ we choose $A_{k} \in \mathscr{L}(\mathscr{E})$ such that $A_{k} a=a_{k}$. Then we obtain

$$
(A a, a) \leqslant\left(\Gamma\left[f_{1}-\sum_{k=1}^{m} A_{k} f_{-k}, f_{1}-\sum_{j=1}^{m} A_{j} f_{-j}\right] a, a\right)=\sum_{k, j=0}^{m}\left(\Gamma\left[f_{j}, f_{k}\right] a_{j}, a_{k}\right) .
$$

Using again (3.4), we see that $A \leqslant \Gamma\left[f_{1}-\hat{f}_{1}, f_{1}-\hat{f}_{1}\right]$.

This propositions shows that if in some way we can determine $\hat{f}_{1}$, then this contains the best information about $f_{1}$ that we can extract from the knowledge of the process up to the moment $t=0$. This justifies calling $\hat{f}_{1}$ the predictible part of $f_{1}$ and $\Delta[f]=\Gamma\left[f_{1}-\hat{f}_{1}, f_{1}-\hat{f}_{1}\right]$ the prediction-error operator.

Now we can formulate more precisely the prediction problems in the following manner:

(1) To determine a sequence of finite operators $\left(A_{1}, \ldots, A_{m}\right)_{(N)}$ in $\mathscr{L}(\mathscr{E})$ such that $\left(\sum_{k} A_{k} f_{-k}\right)_{(N)}$ tends strongly in $\mathscr{L}(\mathscr{E}, \dot{\mathscr{K}})$ to $\hat{f_{1}}$.

(2) To compute the prediction-error operator $\Delta[f]$.

As in the Wiener-Kolmogorov theory of prediction, what is supposed to be known is the correlation function

$$
\Gamma(n)=\Gamma\left[f_{m+n}, f_{n}\right] .
$$

It is clear that $\Gamma(n)$ is an $\mathscr{L}(\mathscr{E})$-valued positive definite function on the group of integers. Using the Naimark dilation theorem, we can represent $\Gamma(n)$ in the form

$$
\Gamma(n)=\int_{0}^{2 \pi} e^{-i n t} d F(t),
$$

where $F$ is an $\mathscr{L}(\mathscr{E})$-valued semi-spectral measure on the undimensional torus, the so called spectral distribution of the process $\left\{f_{n}\right\}_{-\infty}^{+\infty}$. It is easy to verify that $\left[\mathscr{K}_{\infty}^{f}, V_{f}, E\right]$, where $E$ is the spectral measure of the unitary operator $U_{f}^{*}$, is the spectral dilation of $F$. When no confusion can arrise, we denote it by $[\mathscr{K}, V, E]$. In [3] we attached to any $\mathscr{L}(\mathscr{E})$-valued semi-spectral measure $F$ an outer $L^{2}$-bounded analytic function $\{\mathscr{E}, \mathscr{F}, \Theta(\lambda)\}$ which is maximal with the property that its semispectral measure $F_{\theta}$ verifies $F_{\theta} \leqslant F$. (See for details [3].) In [3] and [4] we also proved that

$$
\begin{aligned}
(\Delta[f] a, a) & =\inf _{a_{0}=a, a_{1}, \ldots, a_{n} \in \mathcal{E}} \sum_{k, j=0}^{n} \int_{0}^{2 \pi} e^{i(k-j) t} d\left(F(t) a_{k}, a_{j}\right) \\
& =\inf \sum_{k, j=0}^{n} \int_{0}^{2 \pi} e^{i(k-j) t} d\left(F_{\Theta}(t) a_{k}, a_{j}\right) \\
& =(\Theta(0) * \Theta(0) a, a) .
\end{aligned}
$$

In fact, $F_{\theta}$ is the spectral distribution of the moving average part $\left\{u_{n}\right\}_{-\infty}^{+\infty}$ of $\left\{f_{n}\right\}_{-\infty}^{+\infty}$. We also have: $0 \leqslant \Delta[f] \leqslant \Gamma(0) ; \Delta[f]=0$ iff $\left\{f_{n}\right\}_{-\infty}^{+\infty}$ is deterministic; $\Delta[f]=\Gamma(0)$ iff $\left\{f_{n}\right\}_{-\infty}^{+\infty}$ is a white noise process; $\Delta[f] \geqslant \Delta[p]$ for any white noise process $\left\{p_{n}\right\}_{-\infty}^{+\infty}$ contained in $\left\{f_{n}\right\}_{-\infty}^{+\infty}$; and $\Delta[f]=\Delta[g]$ if $\left\{g_{n}\right\}_{-\infty}^{+\infty}$ is the maximal white noise process contained in $\left\{f_{n}\right\}_{-\infty}^{+\infty}$.

As regards the first part of the prediction problems, to determine the predictible part $\hat{f}_{1}$ of $f_{1}$, it is rather a difficult task. From the formulas $\Gamma\left[\hat{f}_{1}, g_{1}\right]=0$, $\Gamma\left[h, g_{1}\right]=0$ for any $h \in \mathscr{H}_{0}$ and

$$
\Gamma\left[g_{1}, g_{1}\right]=\inf _{h \in \mathscr{H}_{0}} \Gamma\left[f_{1}-h, f_{1}-h\right]
$$

we can interpret $f_{1}=\hat{f}_{1}+g_{1}$ like an orthogonal (in $T$ ) decomposition of $f_{1}$ with respect to $\mathscr{H}_{0}$. From this it results that $\hat{f}_{1}$ is in a sense close to $\mathscr{H}_{0}$, but the problem 
to describe this "closeness" by an approximation procedure seems to be very complicated. However, under some supplementary boundedness condition on the spectral distribution $F$, similar to that imposed by Wiener and Masani in the matrix valued case [8], we shall determine, in the next section, $\hat{f_{1}}$ as the sum (in the strong sense) of an infinite series of elements from $\mathscr{H}_{0}$.

\section{Linear predictor}

The supplementary boundedness condition on $F$ is the following: there exists a constant $c>0$ such that

$$
\frac{1}{2 \pi} c d t \leqslant F \leqslant \frac{1}{2 \pi} c^{-1} d t .
$$

We shall begin with the following

Proposition 4. Let $F$ be an $\mathscr{L}(\mathscr{E})$-valued semi-spectral measure on $T,\{\mathscr{E}, \mathscr{F}$, $\Theta(\lambda)\}$ be its maximal outer function, and $G=\Theta(0) * \Theta(0)$. Then $F$ verifies condition (4.1) if and only if $\{\mathscr{E}, \mathscr{F}, \Theta(\lambda)\}$ is a bounded analytic function which has a bounded analytic inverse, $F_{\Theta}=F, \operatorname{dim} \mathscr{E}=\operatorname{dim} \mathscr{F}$ and there exists an identification of $\{\mathscr{E}, \mathscr{F}, \Theta(\lambda)\}$ with an invertible bounded analytic function $\{\mathscr{E}, \mathscr{E}, \Phi(\lambda)\}$ such that (4.2)

$$
\Phi(0)=G^{1 / 2} \text {. }
$$

Proof. Let $\{\mathscr{E}, \mathscr{E}, \Phi(\lambda)\}$ be identified with $\{\mathscr{E}, \mathscr{F}, \Theta(\lambda)\}$ as in the proposition and let $\{\mathscr{E}, \mathscr{E}, \Psi(\lambda)\}$ be its inverse. Then there exist the Fatou limits $\Phi\left(e^{i t}\right)$ and $\Psi\left(e^{i t}\right)$ and

$$
d F=d F_{\Phi}=\frac{1}{2 \pi} \Phi\left(e^{i t}\right) * \Phi\left(e^{i t}\right) d t .
$$

For any trigonometric polynomial $p$ and $a \in \mathscr{\delta}$ we have

$$
\begin{aligned}
\int_{0}^{2 \pi}\left|p\left(e^{i t}\right)\right|^{2} d(F(t) a, a) & =\frac{1}{2 \pi} \int_{0}^{2 \pi}\left\|\Phi\left(e^{i t}\right) p\left(e^{i t}\right) a\right\|^{2} \\
& \leqslant\|\Phi\|^{2} \frac{1}{2 \pi} \int_{0}^{2 \pi}\left|p\left(e^{i t}\right)\right|^{2}\|a\|^{2} d t
\end{aligned}
$$

and

$$
\begin{aligned}
\int_{0}^{2 \pi}\left|p\left(e^{i t}\right)\right|^{2} d(F(t) a, a) & =\frac{1}{2 \pi} \int_{0}^{2 \pi}\left\|\Phi\left(e^{i t}\right) p\left(e^{i t}\right) a\right\|^{2} d t \\
& \geqslant \frac{1}{2 \pi}\|\Psi\|^{-2} \int_{0}^{2 \pi}\left\|\Psi\left(e^{i t}\right) \Phi\left(e^{i t}\right) p\left(e^{i t}\right) a\right\|^{2} d t \\
& =\frac{1}{2 \pi}\|\Psi\|^{-2} \int_{0}^{2 \pi}\left|p\left(e^{i t}\right)\right|^{2}\|a\|^{2} d t
\end{aligned}
$$

where $\Phi$ and $\Psi$ are the multiplication operators in $\mathscr{L}(\mathscr{E})$ generated by $\Phi\left(e^{i t}\right)$, respectively $\Psi\left(e^{i t}\right)$. It results that for any positive continuous function $\varphi$ on $\boldsymbol{T}$ we have

$$
\frac{1}{2 \pi}\|\Psi\|^{-2} \int_{0}^{2 \pi} \varphi d t \leqslant \int_{0}^{2 \pi} \varphi d F \leqslant \frac{1}{2 \pi}\|\Phi\|^{2} \int_{0}^{2 \pi} \varphi d t,
$$

i.e. $F$ satisfies (4.1).

Conversely, suppose that $F$ satisfies (4.1). If $[\mathscr{K}, V, E]$ is the spectral dilation of $F$ and $U$ is the unitary operator corresponding to $E$, then

$$
X\left(\sum_{n} U^{n} V a_{n}\right)=\sum_{n} e^{i n t} a_{n}
$$

defines an invertible operator from $\mathscr{K}_{+}$to $H^{2}(\mathscr{E})$, which intertwines $U$ with the shift operator on $H^{2}(\mathscr{E})$. Then clearly

$$
X\left(\bigcap_{n \geqslant 0} U^{n} \mathscr{K}_{+}\right)=\bigcap_{n \geqslant 0} U^{n} X \mathscr{K}_{+}=\{0\} .
$$

Thus $\bigcap_{n \geqslant 0} U^{n} \mathscr{K}_{+}=\{0\}$, which implies (by the factorization theorem [3]) that $F_{\Theta}=F$. Obviously, (4.1) implies that $\{\mathscr{E}, \mathscr{F}, \Theta(\lambda)\}$ is bounded and the corresponding $\Theta_{+}$is a bounded operator with bounded inverse $\Theta_{+}^{-1}$. The operator $\Theta_{+}^{-1}$ intertwines the shifts; thus it arises from a bounded analytic function $\{\mathscr{F}, \mathscr{E}, \Omega(\lambda)\}$ which is the inverse of $\{\mathscr{E}, \mathscr{F}, \Theta(\lambda)\}$.

Let us consider the operator $X: \mathscr{F} \rightarrow \mathscr{E}$ defined by $X=G^{1 / 2} \Omega(0)$, where $G$ $=\Theta(0) * \Theta(0)$.

We have

$$
\begin{aligned}
\|X a\|^{2} & =\left\|G^{1 / 2} \Omega(0) a\right\|^{2}=(G \Omega(0) a, \Omega(0) a) \\
& =\left(\Theta(0)^{*} \Theta(0) \Omega(0) a, \Omega(0) a\right)=\|\Theta(0) \Omega(0) a\|^{2}=\|a\|^{2} .
\end{aligned}
$$

Hence $X$ is a unitary operator from $\mathscr{F}$ onto $\mathscr{E}$.

If we put

then we have

$$
\Phi(\lambda)=X \Theta(\lambda), \quad \lambda \in D
$$

$$
\Phi(0)=X \Theta(0)=G^{1 / 2} \Omega(0) \Theta(0)=G^{1 / 2} .
$$

Clearly, $\{\mathscr{E}, \mathscr{E}, \Phi(\lambda)\}$ is another identification of the same function $\{\mathscr{E}, \mathscr{F}, \Theta(\lambda)\}$. The proof is finished.

Let now $\left\{f_{n}\right\}_{-\infty}^{+\infty}$ be a $\Gamma$-stationary process whose spectral distribution $F$ satisfies (4.1). Its prediction-error operator $\Delta[f]=G$ is then an invertible operator on $\mathscr{E}$. Let $\left\{g_{n}\right\}_{-\infty}^{+\infty}$ be the maximal white noise contained in $\left\{f_{n}\right\}_{-\infty}^{+\infty}$. Denote

$$
h_{n}=G^{-1 / 2} g_{n} \text {. }
$$

Then $\left\{h_{n}\right\}_{-\infty}^{+\infty}$ is a white noise process such that

$$
\Gamma\left[h_{n}, h_{n}\right]=I_{\delta} \text {. }
$$

The process $\left\{h_{n}\right\}_{-\infty}^{+\infty}$ is called the normalized innovation process of $\left\{f_{n}\right\}_{-\infty}^{+\infty}$. 
Let $\{\mathscr{E}, \mathscr{E}, \Theta(\lambda)\}$ be the maximal outer function of $F$ identified as in Proposition 4 . Then the geometric model for prediction can be drawn as follows:

$$
\begin{gathered}
\mathscr{K}=L^{2}(\mathscr{E}), \quad \mathscr{K}_{+}=L_{+}^{2}(\mathscr{E}), \\
V=\Theta \mid \mathscr{E}, \quad(V a)(t)=\Theta\left(e^{i t}\right) a,
\end{gathered}
$$

$U=$ the multiplication by $e^{-i t}$ in $L^{2}(\mathscr{E})$.

We have also the following identification for our processes viewed as operators from $\mathscr{E}$ into $L^{2}(\mathscr{E})$ :

$$
\begin{aligned}
& f_{n}: a \rightarrow e^{-i n t} \Theta\left(e^{i t}\right) a, \\
& g_{n}: a \rightarrow e^{-i n t} \Theta(0) a=e^{-i n t} G^{1 / 2} a, \\
& h_{n}: a \rightarrow e^{-i n t} a .
\end{aligned}
$$

Let us write also the Taylor expansions of the function $\{\mathscr{E}, \mathscr{E}, \Theta(\lambda)\}$ and its inverse $\{\mathscr{E}, \mathscr{E}, \Omega(\lambda)\}$ as follows:

$$
\begin{aligned}
& \Theta(\lambda)=G^{1 / 2}+\sum_{k=1}^{\infty} \Theta_{k} \lambda^{k}, \\
& \Omega(\lambda)=G^{-1 / 2}+\sum_{k=1}^{\infty} \Omega_{k} \lambda^{k} .
\end{aligned}
$$

PRoposition 5. Let $\left\{f_{n}\right\}_{-\infty}^{+\infty}$ be a $\Gamma$-stationary process whose spectral distribution $F$ satisfies the boundedness condition (4.1). Then we have

$$
f_{n}=\sum_{k=0}^{\infty} \Theta_{k} h_{n-k}
$$

and

$$
h_{n}=\sum_{k=0}^{\infty} \Omega_{k} f_{n-k}
$$

where the series are supposed to be convergent in the strong topology on $\mathscr{L}(\mathscr{E}, \mathscr{K})$.

Proof. Working with the above identifications, for any $a \in \mathscr{\&}$ we have

$$
\sum_{k=0}^{\infty} \Theta_{k} h_{n-k} a=\sum_{k=0}^{\infty} e^{-i(n-k) t} \Theta_{k} a=e^{-i n t} \sum_{k=0}^{\infty} e^{i k t} \Theta_{k} a=e^{-i n t} \Theta\left(e^{i t}\right) a=f_{n} a
$$

and

$$
\begin{aligned}
\sum_{k=0}^{\infty} \Omega_{k} f_{n-k} a & =\sum_{k=0}^{\infty} e^{-i(n-k) t} \Theta\left(e^{i t}\right) \Omega_{k} a \\
& =e^{-i n t} \Theta\left(e^{i t}\right) \sum_{k=0}^{\infty} e^{i k t} \Omega_{k} a=e^{-i n t} \Theta\left(e^{i t}\right) \Omega\left(e^{i t}\right) a \\
& =e^{-i n t} a=h_{n} a .
\end{aligned}
$$

The convergence of the series and the commutation of the operators with the summations which appeared above is verifiable in an obvious manner.
THEOREM 2. Let $\left\{f_{n}\right\}_{-\infty}^{+\infty}$ be a $\Gamma$-stationary process whose spectral distribution $F$ satisfies the boundedness condition (4.1), $\{\mathscr{E}, \mathscr{E}, \Theta(\lambda)\}$ be the attached maximal outer function and $\{\mathscr{E}, \mathscr{E}, \Omega(\lambda)\}$ be its inverse. Then the predictible part $\hat{f}_{n}$ of $f_{n}$ is given by

where

$$
\hat{f}_{n}=\sum_{j=0}^{\infty} E_{j} f_{(n-1)-j}
$$

The prediction-error operator $\Delta[f]$ is

$$
E_{j}=\sum_{p=0}^{j} \Theta_{p+1} \Omega_{j-p}
$$

$$
\Delta[f]=\Theta(0) * \Theta(0) .
$$

Proof. From (4.7) and (4.8) we obtain

$$
\begin{aligned}
\hat{f}_{n}=f_{n}-g_{n} & =\sum_{k=0}^{\infty} \Theta_{k} h_{n-k}-G^{1 / 2} h_{n}=\sum_{k=1}^{\infty} \Theta_{k} h_{n-k} \\
& =\sum_{k=1}^{\infty} \Theta_{k} \sum_{s=0}^{\infty} \Omega_{s} f_{n-k-s}=\sum_{p=0}^{\infty} \sum_{s=0}^{\infty} \Theta_{p+1} \Omega_{s} f_{n-;} \\
& =\sum_{j=0}^{\infty}\left(\sum_{p+s=j} \Theta_{n+1} \Omega_{s}\right) f_{(n-1)-j}=\sum_{j=0}^{\infty}\left(\sum_{p=0}^{j} \Theta_{p+1} \Omega_{j-p}\right) f_{(n-1)-j} .
\end{aligned}
$$

The convergence of the series and the commutations involved are easily verifiable.

In such a way we can obtain the predictible part $\hat{f}_{n}$ of $f_{n}$ using the linear (infinite) filter $E_{1}, E_{2}, \ldots$, so-called the linear predictor or Wiener filter for prediction.

\section{References}

[1] N. A r o n s z a j n, Theory of reproducing kernels, Trans. Amer. Math. Soc. 68 (1950), 337-404. [2] P. M a s a $\mathrm{n}$ i, An explicit treatment of dilation theory (preprint).

[3] I. S u ciu and I. Valus escu, Factorization of semi-spectral measures, Rev. Roum. Math. pures et appl. 21 (6) (1976), 773-793.

[4] -, -, Essential parameters in prediction, ibid. 22 (10) (1977).

[5] -, -, Fatou and Szegö theorem for operator valued analytic function, Preprint INCREST No. 27 (1977).

[6] B. Sz.-N a g y and C. F o i a s, Harmonic analysis of operators on Hilbert space, Acad. Kiadó, Budapest, North-Holland Publishing Company, Amsterdam-London 1970.

[7] $\mathrm{N}$. W i e $\mathrm{n}$ r and $\mathrm{P}$. M a s a $\mathrm{i}$, The prediction theory of multivariate stochastic processes I, Acta Math. 98 (1957), 111-150.

[8] -, -, The prediction theory of multivariate stochastic processes II, ibid. 99 (1958), 93-139.

\section{Presented to the semester} Spectral Theory

September 23-December 16, 1977 